\title{
Real-time feedback control of the plasma density profile on ASDEX Upgrade
}

\author{
A. Mlynek, M. Reich, L. Giannone, W. Treutterer, K. Behler, H. Blank, A. Buhler, R. Cole, H. Eixenberger, R. \\ Fischer, A. Lohs, K. Lüddecke, R. Merkel, G. Neu, F. Ryter, D. Zasche and the ASDEX Upgrade Team \\ Max-Planck-Institut für Plasmaphysik, 85748 Garching, Germany
}

\begin{abstract}
The spatial distribution of density in a fusion experiment is of significant importance as it enters in numerous analyses and contributes to the fusion performance. The reconstruction of the density profile is therefore commonly done in offline data analysis. In this article, we present an algorithm which allows for density profile reconstruction from the data of the submillimeter interferometer and the magnetic equilibrium in real-time. We compare the obtained results to the profiles yielded by a numerically more complex offline algorithm. Furthermore, we present recent ASDEX Upgrade experiments in which we used the real-time density profile for active feedback control of the shape of the density profile.
\end{abstract}

\section{INTRODUCTION}

For a given number of particles and a given, centrally peaked temperature profile, a fusion device yields higher fusion performance when the spatial distribution of the plasma density is peaked in the center. On the other hand, a too strongly peaked profile has negative effects for magnetohydrodynamical stability and central impurity accumulation. The reconstruction of the density profile is therefore an important step in the analysis of plasma discharges. Profile calculation is often based on the data of several diagnostics, which have their specific properties. Interferometry provides a rather accurate line-integrated measurement of the electron density along a given line of sight through the plasma. The number of chords is usually rather limited as each one requires at least one individual vessel port. An exception are socalled scanning interferometers [1]. Thomson scattering yields a local density measurement, but is limited by the repetition rate of the lasers. Lithium beam impact excitation spectroscopy [2] provides a local density measurement, but is restricted to the plasma edge. Based on the data of such diagnostics and usually with the assumption of constant density on flux surfaces, the profile can be reconstructed. Experiments have shown that in spite of the fact that fuelling is only peripheral in most cases, centrally peaked density profiles are regularly observed in tokamak discharges [3]. The amount of central peaking, however, depends on the plasma parameters and can eventually be influenced by applying local heat sources. On ASDEX Upgrade, the response of the density profile to central electron heating has been extensively studied [4].

In the past few years, real-time diagnostics have been installed on many fusion devices. Those diagnostics process the raw data received from the sensors by evaluation algorithms and make the computed physical quantities available to the discharge control system, which can generate control commands on this basis. This allows for access to advanced operation regimes, which can only be established and sustained by feedback control. The expression 'real-time' refers to the fact that the data received by the control system must be up to date, i.e. the computation of a physical quantity by a real-time diagnostic must be completed within a period shorter than the time scale on which this quantity changes. Due to the importance of the density profile, we have set up a real-time diagnostic on ASDEX Upgrade which calculates the density profile. Whereas profile reconstruction algorithms for offline data analysis can have large numerical complexity, the real-time calculation of a density profile during a plasma discharge is more challenging. In order to meet the realtime requirements and to obtain good time resolution, the calculation has to be completed within a short time interval and the processor speed of the available computers therefore sets an upper limit to the complexity of the algorithm. Real-time algorithms for profile calculation based on the data from magnetic measurements and infrared interferometry and polarimetry have been proposed in the past, in which the profile is parameterized as a $6^{\text {th }}$ degree polynomial of the normalized poloidal flux radius $\rho$, including only even powers of $\rho[5]$, or as a $4^{t h}$ degree polynomial [6].

Here we describe our recent real-time feedback control system for the plasma density profile. We set up a computer algorithm that calculates the density profile from the 5 line-integrated measurements of the submillimeter interferometer by parameterizing the profile as a polynomial and calculating the set of polynomial coefficients providing best agreement with the actually measured line-integrals via least squares approximation. The numerical complexity of the algorithm is low enough to allow for real-time application on present-day computers, but the results are nevertheless in good agreement with the density profiles yielded by more complex offline algorithms. From the profile, a scalar parameter is calculated in real-time which measures the amount of central density peaking and acts as input parameter for the feedback loop. The feedback controller compares this peaking parameter to a preprogrammed setpoint value and switches on or off the electron cyclotron resonance heating $(\mathrm{ECRH})$ system to adjust the peakedness of the density profile to the desired value.

In the second section of this article, we present the realtime algorithm for profile calculation, compare its results to those of an existing offline code and describe its im- 
plementation as a real-time plasma diagnostic. In the third section, the response of the density profile to central ECRH pulses in preparatory feed-forward experiments is discussed, and in the fourth section, the results of the experiments with feedback control of the density profile are presented. Section 5 finally summarizes the results and gives an outlook to experiments envisaged for the near future.

\section{REAL-TIME ALGORITHM FOR DENSITY PROFILE CALCULATION}

The ASDEX Upgrade submillimeter interferometer, which uses a deuterium cyanide (DCN) laser with a wavelength of $195 \mu \mathrm{m}$ as the light source, has a total of 5 lines of sight, which are labeled as 'H-1' - 'H-5' and probe the plasma at different spatial locations. $\mathrm{H}-1$ is a central channel (see figure 1), as the innermost flux surface which is touched by its line of sight has a normalized poloidal flux radius $\rho$ of approximately 0.2 for a typical discharge. H-2 and H-3 are situated rather symmetrically around the magnetic axis and both reach a minimum $\rho$ around 0.4 , whereas $\mathrm{H}-4$ and $\mathrm{H}-5$ are edge channels. The interferometer yields a line-integrated measurement of the electron density $n_{e}$ along those 5 chords through the plasma. By assuming constant density on flux surfaces (i.e. $n_{e}=n_{e}(\rho)$ ) and taking into account the reconstructed magnetic equilibrium, it is possible to reconstruct the density profile from the line-integrated measurements. There are several offline algorithms for density profile calculation available on ASDEX Upgrade, which partially include also data from other diagnostics. In the following discussion, the density profiles provided by integrated data analysis (IDA) [7] of the data from interferometry and lithium impact excitation spectroscopy will be used as reference to assess the quality of the profiles calculated by the real-time algorithm.

For the real-time approach, we parameterize the density profile inside the separatrix as a polynomial and assume an exponential decay of density in the scrape-off layer. As the density profile has to be continuously partially differentiable at the magnetic axis when written as a function of the Cartesian coordinates, which will be labelled $\mathrm{R}$ and $\mathrm{z}$ (see figure 1), we have to postulate that the polynomial has zero derivative at $\rho=0$, i.e. there must be no linear term. At the separatrix, our only matching condition is that the density profile is continuous, but we allow for a discontinuity of the first and all higher derivatives. The decay length of the exponential function in the scrape-off layer is calculated from the distance between the separatrix and the limiter and therefore not a free parameter in the reconstruction. Accordingly, we have the following parameterization of the density profile:

$$
n_{e}(\rho)= \begin{cases}\sum_{k=1}^{M} a_{k} f_{k}(\rho) & \text { if } \rho \leq 1 \\ e^{-\lambda(\rho-1)} \cdot \sum_{k=1}^{M} a_{k} f_{k}(1) & \text { if } 1<\rho \leq \rho_{\text {lim }} \\ 0 & \text { if } \rho>\rho_{\text {lim }}\end{cases}
$$

where $\rho_{\text {lim }}$ is the flux radius corresponding to the limiter and the $f_{k}(\rho)$ are general basis functions. In our polynomial approach, we set $f_{1}=1$ and $f_{2}=\rho^{2}$, avoiding a linear term.

Based on the magnetic equilibrium, forward-modelling is done, i.e. the 5 line-integrals corresponding to the interferometer chords are calculated as a function of the profile coefficients $a_{1}, \ldots, a_{M}$. On ASDEX Upgrade, an equilibrium obtained by numerically solving the GradShafranov equation [8] is available for off-line data analysis, but could not yet be run in real-time during the last experimental campaign, as it still consumed too much computing power. Therefore, a functional parameterization approach [9] has been chosen for real-time applications, which provides the normalized poloidal flux radius $\rho$ on a discrete $69 \times 39$ grid with a spacing of $4 \mathrm{~cm}$. This grid is illustrated in figure 1 . We discretize the line in-

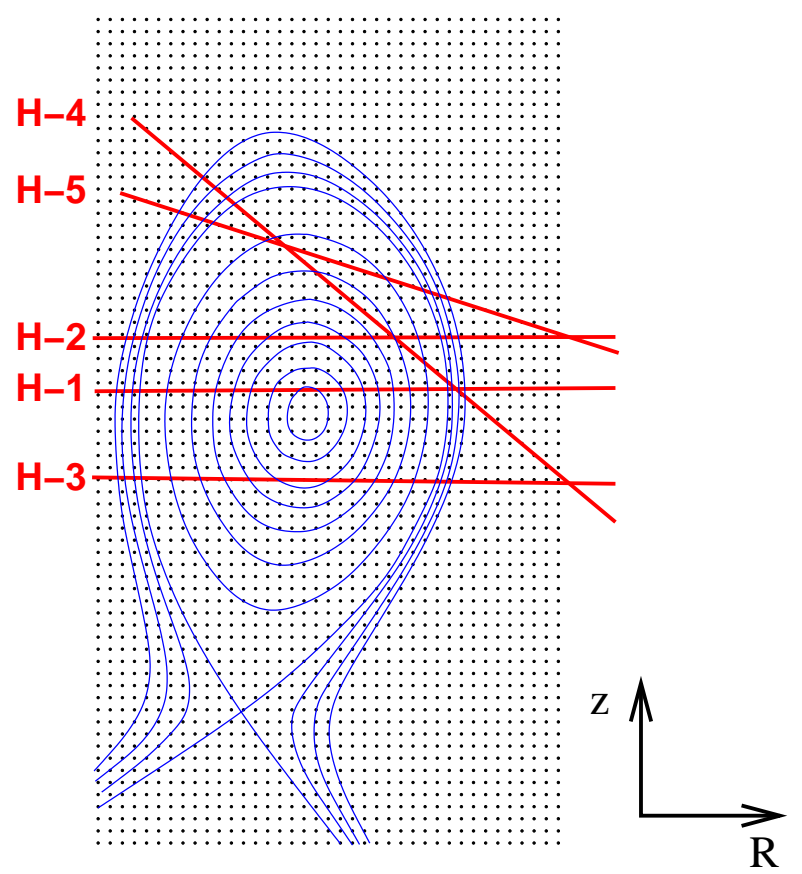

FIG. 1: An illustration of the discrete $69 \times 39$ grid on which the value of $\rho$ is calculated via functional parameterization in real-time. In addition, the lines of sight $\mathrm{H}-1$ to $\mathrm{H}-5$ of the DCN interferometer are shown, as well as some flux surfaces for a typical ASDEX Upgrade discharge.

tegrals along the interferometer chords according to the given grid. We denote the coordinate along a line of sight 
as the $\mathrm{x}$-direction. Via linear interpolation in the vertical direction, the poloidal flux is calculated for a set of equidistant points on the line of sight, see figure 2 . The corresponding values are labelled $\rho_{H, 1}, \ldots, \rho_{H, N}$, where the first index $H \in\{1, \ldots, 5\}$ denotes the interferometer channel $\mathrm{H}-1$ to $\mathrm{H}-5$, and the distance between the points is labelled $d_{H}$. We assume $\rho(x)$ to be linear between those points. Accordingly, the line integral for the $H^{t h}$ line of sight (LOS) can be discretized as

$\int_{L O S H} n_{e}(\rho(x)) d x \doteq \sum_{i=1}^{N-1} \frac{d_{H}}{\rho_{H, i+1}-\rho_{H, i}} \int_{\rho_{H, i}}^{\rho_{H, i+1}} n_{e}(\rho) d \rho$.

If $\rho_{H, i+1}-\rho_{H, i}$ is small, which regularly happens when the minimum of $\rho$ for the given line of sight is enclosed between the two points, this approach is numerically unstable. We therefore replace the summand by $d_{H} n_{e}(\bar{\rho})$ with $\bar{\rho}=\frac{1}{2}\left(\rho_{H, i+1}+\rho_{H, i}\right)$ when the difference $\rho_{H, i+1}-\rho_{H, i}$ is below a certain threshold value.

We perform forward modelling and insert equation (1) into (2). As our parameterization of $n_{e}(\rho)$ is defined in sections, we have to split the line integral into a contribution from the scrape-off layer and a contribution from the main plasma inside the separatrix. When sorting the result by coefficients $a_{1}, \ldots, a_{M}$ and factoring them out, it can be written as

$$
\int_{L O S H} n_{e}(\rho(x)) d x=\sum_{k=1}^{M} a_{k} \beta_{H, k}, \quad H \in\{1, \ldots, 5\}
$$

where the terms $\beta_{H, k}, H \in\{1, \ldots, 5\}$ and $k \in$ $\{1, \ldots, M\}$, represent the (discretized) line integral of the $k^{t h}$ basis function along the $H^{t h}$ line of sight in the main plasma, plus a contribution from the scrape-off layer that includes the exponential decay term. Equation (3) shows that the modelled line integral is a linear combination of the profile coefficients $a_{1}, \ldots, a_{M}$. In the next step, the coefficients $a_{1}, \ldots, a_{M}$ are determined via least squares approximation to provide best consistency between the modelled and the measured line integrals. We assume that the line integrated densities are measured with stochastic errors $\epsilon_{H}$ with standard deviations $\sigma_{H}$. The measured line integrals are labelled $L_{H}$, $H \in\{1, \ldots, 5\}$. Then we have

$$
L_{H}=\int_{L O S H} n_{e}(\rho(x)) d x+\epsilon_{H} .
$$

The sum $\chi^{2}$ of the squares of the errors, normalized to the standard deviations, is then given by

$$
\chi^{2}=\sum_{H=1}^{5} \frac{\left(L_{H}-\sum_{k=1}^{M} a_{k} \beta_{H, k}\right)^{2}}{\sigma_{H}^{2}} .
$$

The profile that agrees best with the measurements minimizes this $\chi^{2}$. A necessary condition for that requires the partial derivatives with respect to the profile parameters to vanish, i.e.

$$
\frac{\partial \chi^{2}}{\partial a_{j}}=2 \sum_{H=1}^{5} \frac{\left(L_{H}-\sum_{k=1}^{M} a_{k} \beta_{H, k}\right)\left(-\beta_{H, j}\right)}{\sigma_{H}^{2}} \stackrel{!}{=} 0 \quad \forall j \in\{1, \ldots
$$

We now introduce matrix-vector notation by defining the 5 -vector $\vec{l}$ which contains $L_{1}, \ldots, L_{5}$, the M-vector $\vec{a}$ containing the profile coefficients $a_{1}, \ldots, a_{M}$, the $5 \times M$ matrix $B$ and the $M \times 5$ matrix $\Gamma$ with

$$
(B)_{H k}=\beta_{H, k} \quad \text { and } \quad(\Gamma)_{j H}=\frac{\beta_{H, j}}{\sigma_{H}^{2}} .
$$

Then, all $M$ equations in (6) can be written as one matrix-vector-equation:

$$
\Gamma \cdot(\vec{l}-B \cdot \vec{a})=\overrightarrow{0} .
$$

If $\Gamma B$ is not singular, the solution is given by

$$
\vec{a}=(\Gamma \cdot B)^{-1} \cdot \Gamma \cdot \vec{l}
$$

When the number of free parameters is equal to the number of interferometer chords, $B$ and $\Gamma$ are square matrices, so the identity $(\Gamma \cdot B)^{-1}=B^{-1} \cdot \Gamma^{-1}$ holds and one gets $\vec{a}=B^{-1} \cdot \vec{l}$, i.e. all measured line integrals can be precisely reproduced. Due to the symmetry between interferometer chords $\mathrm{H}-2$ and $\mathrm{H}-3$ (see figure 1 ), their line integrals are similar and the corresponding lines in the matrix $B$ do not differ much, resulting in a poor condition number. Therefore, the use of 5 free parameters is unfavourable.

We performed calculations with 2, 3 and 4 free parameters. By comparing the results to those provided by the offline algorithm IDA, we found that the use of only 2 free parameters does not allow for a reasonable description of the full variety of different profile shapes observed in typical plasma discharges. When using 4 free parameters, there is good agreement with IDA in many cases, but sometimes, the same problem as described in [5] is observed: The profile is non-monotonic in the plasma edge, with local minima around $\rho=0.8$. Best results were obtained with 3 free parameters. This can be understood from the chord geometry of the interferometer: There is not only a symmetry between H-2 and H-3, but also the edge channels H-4 and H-5 probe a similar part of the plasma. Depending on the location of the flux surfaces, the minimum $\rho$ of their lines of sight can be similar, which makes the inversion problem numerically unstable and causes oscillations at the edge.

When using 3 free parameters, the function $a_{1}+a_{2} \rho^{2}+a_{3} \rho^{3}$ was our first choice, i.e. the basis functions were $f_{1}=1, f_{2}=\rho^{2}$ and $f_{3}=\rho^{3}$. However, 


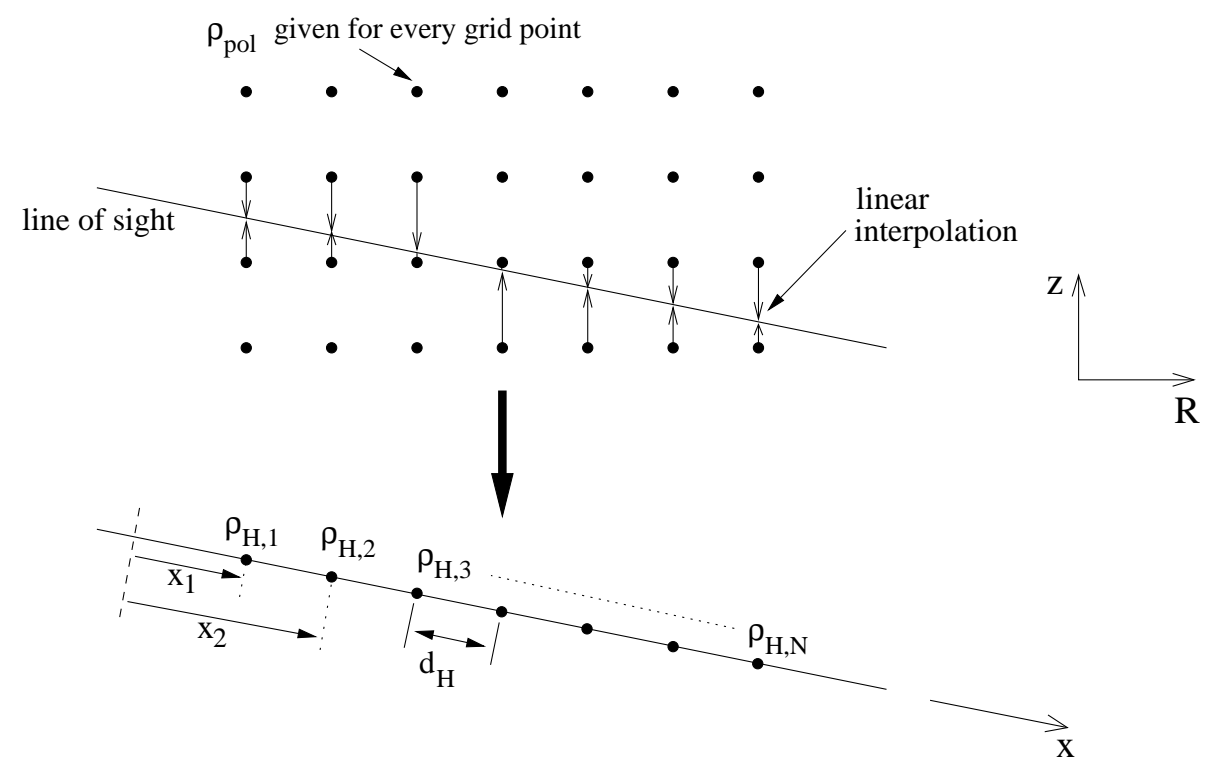

FIG. 2: The poloidal flux is calculated for equidistant points on the line of sight via linear interpolation.

this method was found not to provide a good description of centrally peaked density profiles. We therefore replaced the third basis function by $f_{3}=\rho^{3}+\mu \rho^{4}$ and set $\mu$ to a fixed value (-0.452), which was found to allow for a realistic description also of peaked profiles. Figure 3 compares the results provided by the real-time algorithm with these settings to the IDA profiles for two different discharges. Evidently, deviation is mainly observed at the edge. This can be understood from the fact that in contrast to IDA, the real-time code does not make use of the data from lithium beam impact excitation spectroscopy. The lithium beam diagnostic is not yet delivering data in real-time and operates with a pulsed beam with off-periods of $24 \mathrm{~ms}$. Therefore, it was not included in the real-time profile reconstruction. As the profiles calculated by both algorithms have to match the same 5 line-integrals, a deviation in density at the edge has to be compensated by a deviation with opposite sign further inside the plasma, as the left diagram illustrates. Figure 4 shows a systematic comparison of the local density provided by the real-time algorithm and IDA at 4 different radii, including data from 50 different plasma discharges and covering a large range of density (more than one order of magnitude). One observes good agreement of the two density profiles at $\rho=0.2$ and $\rho=0.5$. Towards the edge, the scatter diagrams broaden significantly, which can be seen clearly at $\rho=0.95$. As discussed before, this is due to the edge data from the lithium beam diagnostic which the real-time algorithm does not use. A closer look at the top left diagram $(\rho=0.2)$ reveals that the scatter slightly increases towards low density. This can be understood from the fact that the innermost flux surface probed by the central interferometer channel $\mathrm{H}-1$ usually has a flux radius around $\rho=0.2$, so that the density reconstructed for $\rho=0.2$ is based on an actual measurement, but had a flux radius close to 0.3 in some of the low-density discharges used for the diagram. In that case, the density reconstructed for $\rho=0.2$ has some extrapolation character and thus shows more deviation between the two algorithms. All in all, the comparison shows that the real-time algorithm provides a density profile that is well adequate for deriving global plasma parameters such as the amount of central density peaking. However, one has to be aware that the edge density is modelled in a simplified way, with the pedestal top always being located at $\rho=1$. Therefore, it is not possible to reliably extract plasma edge characteristics such as the exact location and width of the edge pedestal and the edge density gradient from the real-time profile.

After the development of this algorithm for profile calculation, which mainly consists of the calculation of the $B$ matrix from the magnetic equilibrium, matrix inversion and matrix-vector multiplication, it was implemented on a real-time computer network. The input data for the profile algorithm is the $69 \times 39$ matrix containing the magnetic equilibrium, the flux radius at the limiter, $\rho_{\text {lim }}$, and the 5 line-integrated densities measured by the interferometer, which are validated by a real-time algorithm [10] to reject measurement errors, so called 'fringe jumps'. One therefore needs real-time access to the output of two different diagnostics, namely magnetics and interferometer, which are physically separated and have individual computers. The transfer of the large magnetic equilibrium matrix containing 2691 floating point numbers within a millisecond is challenging and expected to be achieved in the near future with the help of a reflective shared memory technology. In the 2009 experimental campaign, how- 

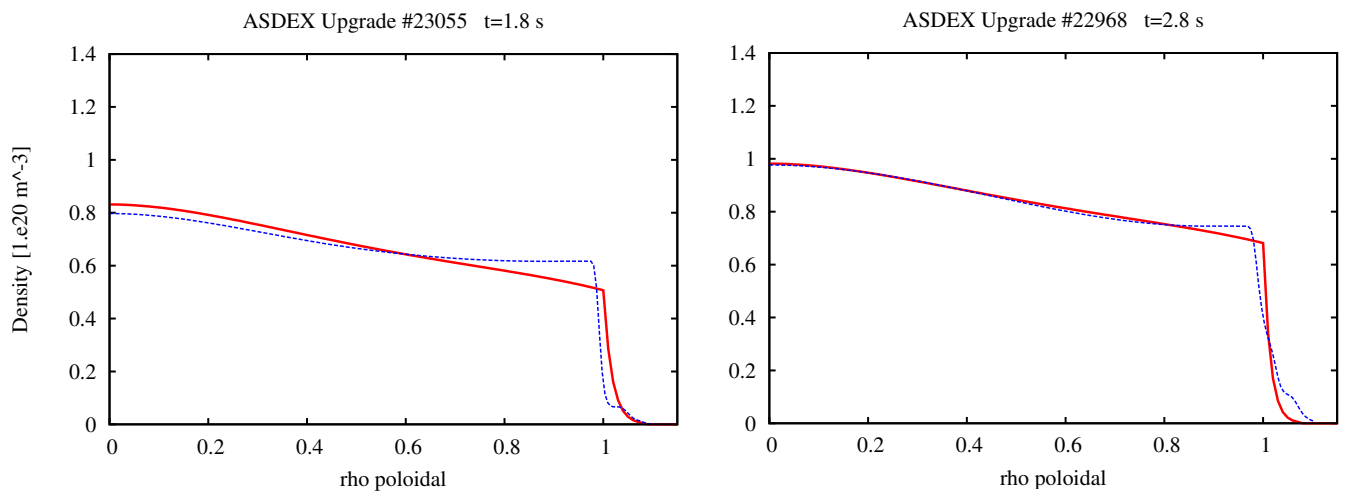

FIG. 3: Comparison of the density profile calculated by the real-time algorithm (bold solid line) and the IDA algorithm (dashed line) for two different discharges.
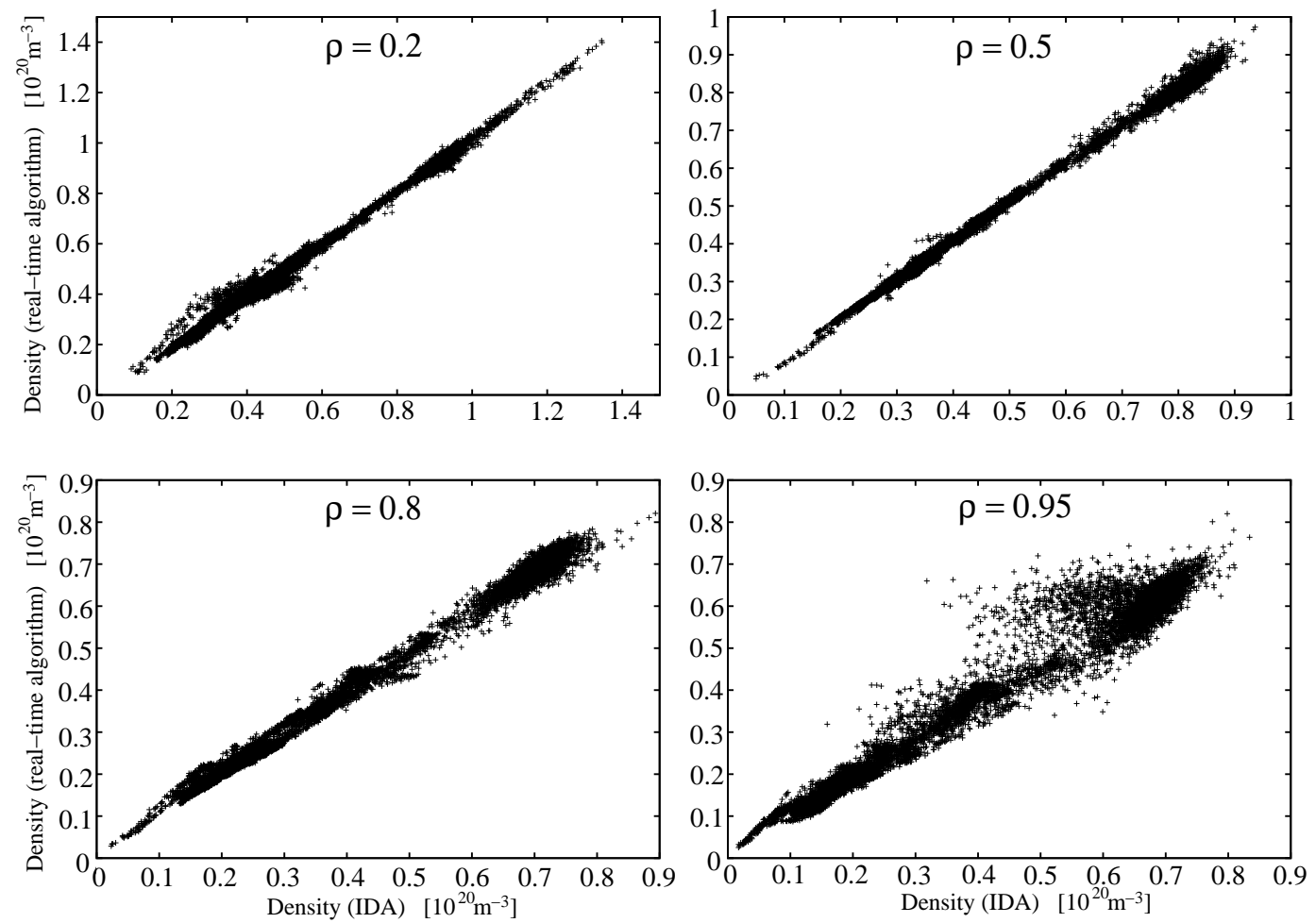

FIG. 4: Comparison of the local density calculated by the IDA algorithm (abscissa) and the real-time algorithm (ordinate) at 4 different radii (top left: $\rho=0.2$, top right: $\rho=0.5$, bottom left: $\rho=0.8$, bottom right: $\rho=0.95$ ). The diagrams were generated from the data of 50 plasma discharges, including both, L-mode and H-mode, and showing one data point each 10 milliseconds.

ever, the computer of the interferometer was connected to the other real-time diagnostics via Ethernet only. With the user datagram protocol (UDP), data exchange between diagnostics was possible on a time scale of less than a millisecond [11], however, the bandwidth was far not sufficient for the transfer of 2691 floating point numbers. This problem was finally overcome by exploiting a rather convenient property of the real-time algorithm: The calculation of the matrix $B$ from the large $69 \times 39$ flux matrix only requires the coordinates of the 5 lines of sight of the interferometer, which are static, but no density measurements. We therefore split the algorithm and implemented this first part on the computer of the magnetic diagnostic. When using 4 free parameters for profile reconstruction, which is the maximum we took into account, $B$ is a $5 \times 4$ matrix. 
Together with the flux radius at the limiter $\rho_{\text {lim }}$, which is also needed for profile calculation, this results in a total of 21 floating point numbers that have to be transferred to the computer of the interferometer. This is within the capabilities of the UDP connection. The second part of the algorithm was implemented on the interferometer side. It calculates the density profile via least squares approximation from the 5 line-integrated density measurements which are obtained from the local interferometer hardware, and from the data received via network. The resulting density profile coefficients are then transferred to the discharge control system. The density profile is calculated each millisecond, as the cycle time of the discharge control system is of this order.

\section{IMPACT OF CENTRAL ELECTRON HEATING ON THE DENSITY PROFILE}

As a first application of real-time density profiles, we performed feedback control of the shape of the profile. For this purpose, an actuator was required that is capable of modifying the spatial distribution of density. The only available non-peripheral fuelling source would have been the injection of pellets. However, when pellets are injected, there is a considerable probability for the interferometer to suffer from fringe jumps, which would have forced us to stop profile calculation. For this reason, we had to select a different actuator for the feedback loop. Modifying the gas puff rate from the plasma edge also influences the density profile. However, as we intended to keep the volume averaged density approximately constant during the feedback control phase, there was hardly any freedom to modify the gas inlet rate.

We have finally chosen central electron cyclotron heating as actuator. Although ECRH in the first instance affects the temperature, also the density profile shows response to central heating under certain conditions through a coupling mechanism between temperature and density. In experiments on ASDEX Upgrade and other tokamaks, it has been shown that central heating results in a reduction of the core density for low-density L-mode discharges [4] [12] [13]. This phenomenon is commonly referred to as density 'pump-out' in the literature. When going towards higher density in L-mode, this effect is reported to become weaker and finally reverse sign. In H-mode, a small but still significative response of the density profile to ECRH is reported for low and high densities, whereas at intermediate density, there is no effect [4].

We performed several preparatory experiments with preprogrammed ECRH pattern in order to define the operational window and the controller parameters for the discharges with feedback controlled density profile. As the feedback control loop requires a scalar input parameter that describes the shape of the density profile, we define the density peaking parameter $p$ as:

$$
p=\frac{\int_{0}^{0.4} n_{e}(\rho) d \rho-\int_{0.6}^{1.0} n_{e}(\rho) d \rho}{\int_{0}^{1} n_{e}(\rho) d \rho}
$$

This parameter is equal to zero for a flat profile, positive for a centrally peaked profile and negative for a hollow profile. In this regard, it differs from other peaking parameters that can be found in the literature (like the ratio of core density and volume averaged density), which are equal to 1 for a flat profile. As the profile is parameterized as a polynomial inside the separatrix, the integration is trivial and the calculation of the peaking parameter therefore does not consume much time. When the density is low, like in the ramp-up phase of the discharge, the denominator is close to zero, making the result obtained for $p$ unstable. Therefore, the peaking parameter has to be labelled as invalid when the total density is below a certain threshold.

In the preparatory experiments, we injected a series of ECRH pulses with a duration of 100 milliseconds and a spacing of $100 \mathrm{~ms}$ into plasmas with a plasma current of $600 \mathrm{kA}$ at different densities. The ECRH beam launcher was adjusted to provide central power deposition. During the phase with the heating pulses, we mostly controlled the gas inlet valves with a feedback loop on the line-integrated density measured by the central interferometer chord H-1. Just for a few sequences of ECRH pulses, we set the gas level to a fixed value to exclude secondary effects resulting from the gas feedback. Figure 5 shows the time traces of the 5 interferometer channels, the applied ECRH power, the gas inlet rate and the peaking parameter $p$ for an L-mode discharge with a lineaveraged density of $2.5 \cdot 10^{19} \mathrm{~m}^{-3}$ (H-1). A clear density pump-out effect is observed, the core density decreases when ECRH is switched on. Due to the line-integrated density feedback on channel $\mathrm{H}-1$, this results in an opening of the gas inlet valves, which in turn makes the edge density rise. Both, decreasing core and rising edge density result in a reduction of the peaking parameter. For both, an ECRH power of about $0.8 \mathrm{MW}$ and $1.5 \mathrm{MW}$, the peaking parameter rises and falls in phase with the heating pulses. Figure 6 shows the density profiles obtained from the IDA algorithm for two cases without additional heating and for cases with 0.8 and $1.5 \mathrm{MW}$ of ECRH. We also carried out discharges with longer heating pulses and with a fixed gas inlet rate to study the pure effect of ECRH. It was found that the peaking parameter then reaches the same level of saturation, it just takes some tens of milliseconds longer until it arrives at this level. Operating with feedback on the line-integrated density therefore speeds up the flattening process, but does not affect the achievable amount of flattening.

We performed a density scan in L-mode which revealed that the pump-out effect is strong up to line-averaged densities of about $2.5 \cdot 10^{19} \mathrm{~m}^{-3}$. In those discharges, the 


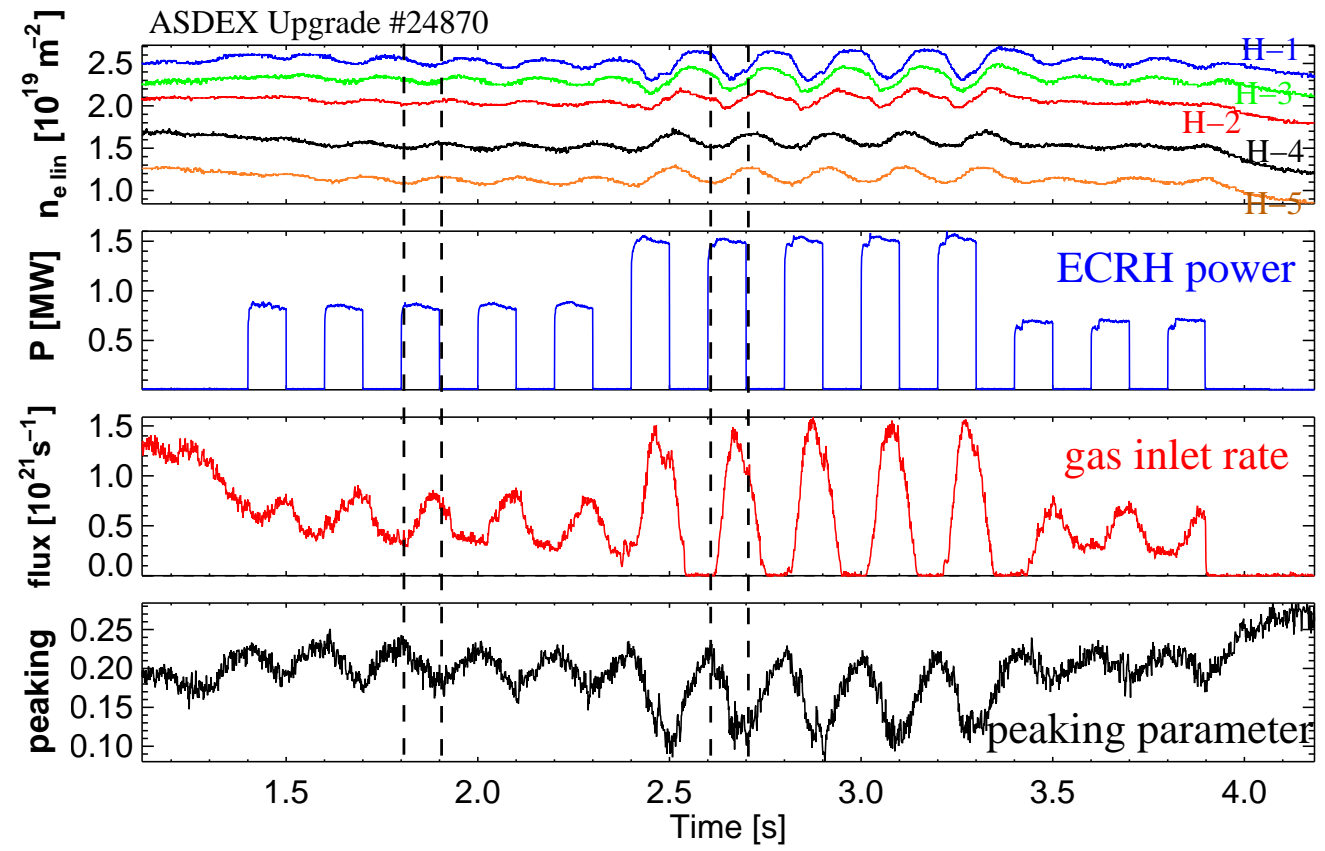

FIG. 5: Density response to ECRH pulses in a $600 \mathrm{kA} \mathrm{L-mode} \mathrm{discharge} \mathrm{at} \mathrm{a} \mathrm{line-averaged} \mathrm{density} \mathrm{of} 2.5 \cdot 10^{19} \mathrm{~m}^{-3}$. The upper box shows the time traces of the 5 interferometer channels, the second box the applied ECRH power, the third one the deuterium inlet rate and the fourth one the peaking parameter $p$.

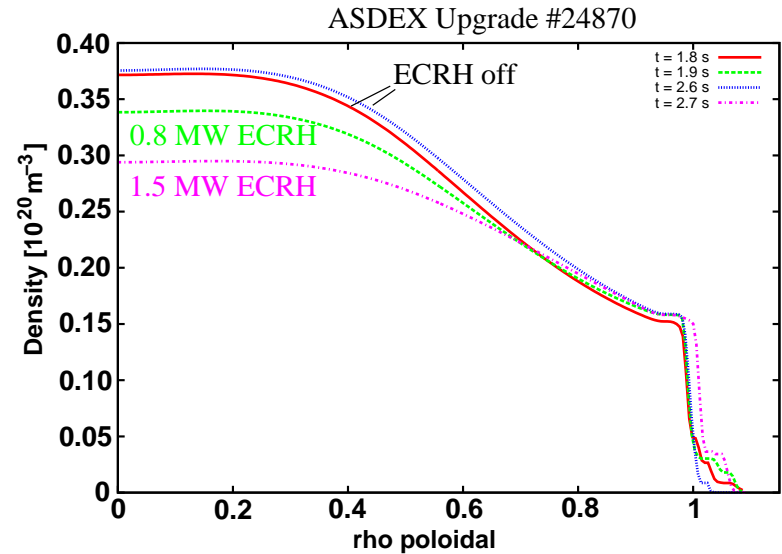

FIG. 6: Density profiles obtained from the IDA algorithm for the 4 points in time which are marked as dashed vertical lines in figure 5 . The solid curve corresponds to $t=1.8 \mathrm{~s}$, the dashed one to $\mathrm{t}=1.9 \mathrm{~s}$, the dotted one to $\mathrm{t}=2.6 \mathrm{~s}$ and the dashdotted one to $\mathrm{t}=2.7 \mathrm{~s}$.

effective collisionality $\nu_{\text {eff }}=0.1 Z_{\text {eff }}\left\langle n_{e}\right\rangle R /\left\langle T_{e}\right\rangle^{2}[14]$ where $\left\langle n_{e}\right\rangle$ and $\left\langle T_{e}\right\rangle$ are the volume average of electron density (in $10^{19} \mathrm{~m}^{-3}$ ) and temperature (in $\mathrm{keV}$ ), is between 0.04 and 0.3 and the dominant turbulence type is a trapped electron mode (TEM). It is known that the
TEM is stabilized at collisionalities above $\nu_{\text {eff }} \approx 0.5$ and an ion temperature gradient (ITG) mode becomes the dominant turbulence type [15]. At $3.0 \cdot 10^{19}$, the pumpout effect is still present, but weaker, and at a density of $4.0 \cdot 10^{19} \mathrm{~m}^{-3}$, where we have $\nu_{\text {eff }}$ between 0.5 and 1 and thus a dominant ITG mode, ECRH has the opposite effect: The core density increases and accordingly, the peaking parameter increases. This is consistent with the observations presented in [4] and explained in [14]. We also performed experiments in $\mathrm{H}$-mode discharges. For many sets of plasma parameters, there was no significant effect of central ECRH on the density profile. The density pump-out in low-density H-mode discharges reported in [4] could not be reproduced as the low level of collisionality present in those previous experiments was no longer accessible on ASDEX Upgrade in the 2009 campaign as the machine has meanwhile been equipped with an all-tungsten first wall. But we finally found a discharge scenario in which ECRH has remarkable influence on the spatial distribution of density in an H-mode discharge. At a plasma current of $600 \mathrm{kA}$, a line-averaged density around $5.0 \cdot 10^{19} \mathrm{~m}^{-3}$ and $2.5 \mathrm{MW}$ of neutral beam heating, ECRH was found to cause a strong increase of the core density, whereas the edge density remains unaffected. Figure 7 summarizes the observed response of the peaking parameter $p$ to ECRH pulses at different densities in the preparatory experiments. 
To sum up, central ECRH reliably causes a reduction of

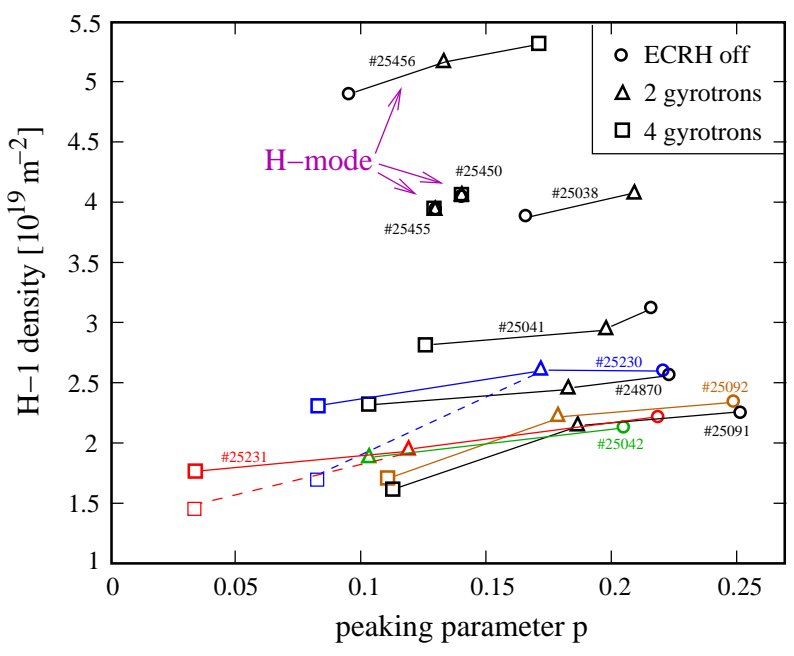

FIG. 7: Response of the peaking parameter $p$ to ECRH pulses. The diagram shows the line-integrated density measured by interferometer channel $\mathrm{H}-1$ and the peaking parameter for the cases without ECRH (circles), with 0.7-0.9 MW of ECRH (triangles) and with 1.5 MW of ECRH (squares). Symbols corresponding to the same discharge are connected by lines. Some discharges were partially performed with and without feedback on the line-integrated $\mathrm{H}-1$ density. In cases where the densities in both phases differ significantly, both data points are shown. The points corresponding to a fixed gas level are then connected by a dashed line.

the core density over a wide range of plasma parameters in low-density L-mode discharges. When going towards higher collisionality, the effect reverses sign and makes the profile more peaked in the center. In H-mode, in contrast, central heating has no effect on the density profile in many cases. Nonetheless, some plasma parameters exist for which central heating makes the profile more peaked. Based on this knowledge, we then performed active feedback control of the shape of the density profile in both, L-mode and $\mathrm{H}$-mode discharges.

\section{EXPERIMENTS WITH ACTIVE CONTROL OF THE DENSITY PROFILE}

For the feedback control experiments, the peaking parameter $p$ as defined in equation (8) was calculated in real-time from the density profile and transferred to the discharge control system via the UDP connection. During the feedback control phase, which had to be restricted to a time window of 2 seconds to ensure that the load limit of the ECRH system is not exceeded, two density controllers were active at the same time: On the one hand, the gas inlet valves were controlled by a feedback loop on the line-integrated density measured by interferometer channel H-1, and on the other hand, the ECRH system was controlled by a feedback loop on the peaking parameter $p$. A time-dependent setpoint for $p$ was defined in the discharge programme. When the peaking parameter received from the interferometer exceeds the setpoint value, the controller turns on ECRH, and when it falls below it, it turns off ECRH. To avoid too frequent switching of the gyrotrons, an additional hysteresis of \pm 0.005 was introduced. With those settings, we performed active profile control first in several L-mode discharges at different density. One example is shown in figure 8 , where we operated at a line-averaged density of $3.0 \cdot 10^{19} \mathrm{~m}^{-3}$ and programmed a linear ramp from 0.09 to 0.2 as the setpoint for $p$. As discussed in the previous section, the density pump-out effect at this density is rather weak. However, when applying 1.5 MW of ECRH power for some tens of milliseconds, a transition to a weakly developed $\mathrm{H}$-mode regime occurs, which results in increasing pedestal density and thus in a reduction of the peaking parameter $p$. The peaking controller was active in the time window from $\mathrm{t}=1.39 \mathrm{~s}$ to $3.29 \mathrm{~s}$. The feedback scheme was successful, the peaking parameter actually oscillates around the setpoint, following the ramp. In the early phase, when the peaking setpoint is around 0.10 , the duty cycle of ECRH modulation is close to 1 . When ECRH is switched off, the peaking parameter immediately starts rising and crosses the upper threshold within approximately 10 milliseconds. Accordingly, ECRH is switched on again, but the peaking parameter nevertheless continues rising at reduced slope for further $50-60 \mathrm{~ms}$. Only then, the previously described transition to H-mode occurs and $p$ starts decreasing. Later in the discharge, when the peaking setpoint is higher, this is different. There, ECRH causes a prompt drop of the peaking parameter. The amplitude of the oscillation around the setpoint is then determined by the controller hysteresis, the latency of the controller, the power rampup time of the gyrotrons and the response time of the plasma to the modified heating power. Figure 8 in addition shows the time trace of channel 55 of the electron cyclotron emission (ECE) radiometer, which probes the plasma at about $\rho=0.25$. The ECE data shows that there is a clear variation of temperature during the feedback control phase, and the transition to $\mathrm{H}$-mode about $50-60 \mathrm{~ms}$ after the beginning of a heating pulse becomes apparent by a sudden increase of the ECE signal.

This discharge was still performed with a density profile and a peaking parameter calculated on the basis of a static equilibrium. By the end of the 2009 experimental campaign, however, the real-time transfer of the $B$ matrix from the magnetic diagnostic to the interferometer had been set up, allowing for changes of the plasma configuration during the shot. We made use of these new possibilities by performing a discharge with a transition from L-mode to $\mathrm{H}$-mode and density profile control in both phases. In the H-mode case, we used the discharge parameters for which a peaking of the density profile due to central ECRH had been identified in the preparatory experiments. This resulted in the need to reverse the control scheme after the transition to H-mode. There, ECRH is turned on when $p$ is below the setpoint and 


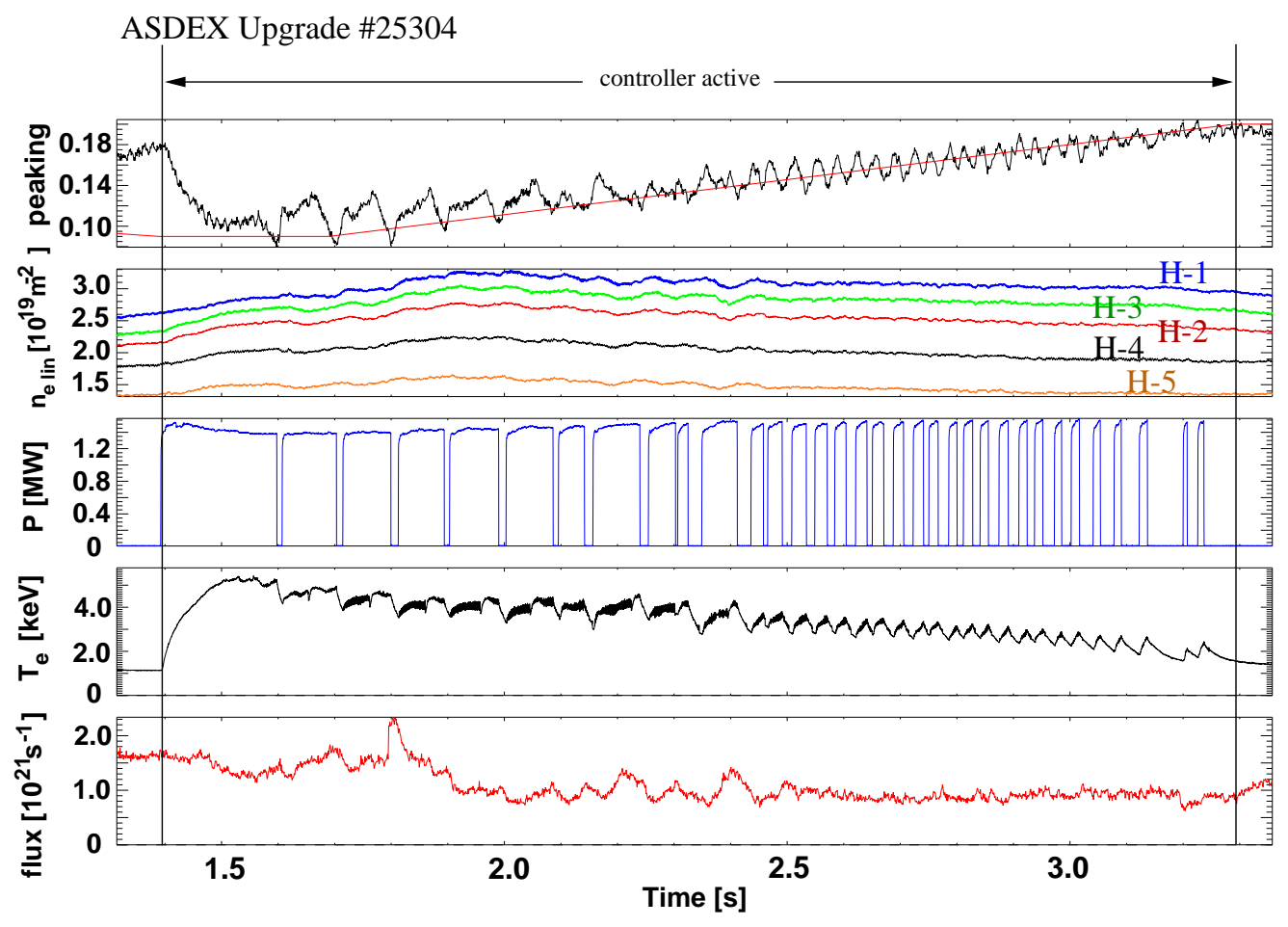

FIG. 8: Feedback control of the density peaking in an L-mode discharge: The diagram shows the time traces of the peaking parameter $p$, including the controller setpoint (red line, hysteresis \pm 0.005 ), the 5 line-integrated densities measured by the DCN interferometer, the applied ECRH power, the electron temperature measured by ECE channel 55 (located at $\rho \approx 0.25$ in this discharge) and the throughput of the gas inlet valves (from top to bottom).

off when it is above. Figure 9 shows the time traces for this discharge. It can be seen that the noise level of the calculated peaking parameter is higher than in the previously discussed discharge, where it was solely determined by the accuracy level of the line-integrated density measurement by the interferometer. Now, density profile calculation is based on the equilibrium provided by a second real-time diagnostic and accordingly, also the uncertainty of equilibrium reconstruction enters into the result. The controller was intended to be active in L-mode configuration from 1.5 to $2.5 \mathrm{~s}$ and in $\mathrm{H}$-mode configuration from 3.3 to $4.3 \mathrm{~s}$. Accordingly, three linear ramps were programmed as setpoint for the density peaking in those two time intervals. For technical reasons, the controller could only be enabled at $t=1.8 \mathrm{~s}$. That way, feedback control in L-mode was restricted to the time window from $1.8-2.5 \mathrm{~s}$. When the controller is switched on, there is a fast response of the peaking parameter, it reaches the setpoint value within about 70 milliseconds, and then follows the setpoint trajectory until $t=2.5 \mathrm{~s}$. After the transition to H-mode, which was performed between 2.5 and $3.3 \mathrm{~s}$, the controller was switched on again for one second. It can be seen that also in H-mode, feedback control was successful as the peaking parameter follows the programmed setpoint.

\section{SUMMARY AND OUTLOOK}

We have developed and installed a network of real-time diagnostics on ASDEX Upgrade, which was operational during the 2009 experimental campaign. On this network, we have implemented a fast algorithm for the realtime calculation of the plasma density profile from the data of the submillimeter interferometer and the magnetic diagnostic. As central ECRH causes a change of the density profile via a coupling mechanism between temperature and density in many cases, we could demonstrate the feasibility of active control of the shape of the density profile with a feedback loop using central ECRH as actuator. The direction in which the profile can be modified depends on the plasma parameters. In lowdensity L-mode discharges, the profile can be flattened compared to the purely ohmic case, whereas at higher density, it can be made more peaked. In H-mode, where low values of $\nu_{\text {eff }}$ were not accessible in the 2009 experiments, the possibilities of this method of profile control are limited by the lack of a response of the density to central heating, which was found for a large range of plasma parameters. Nevertheless, for some H-mode cases, a density response to ECRH exists and feedback control was successfully applied. 


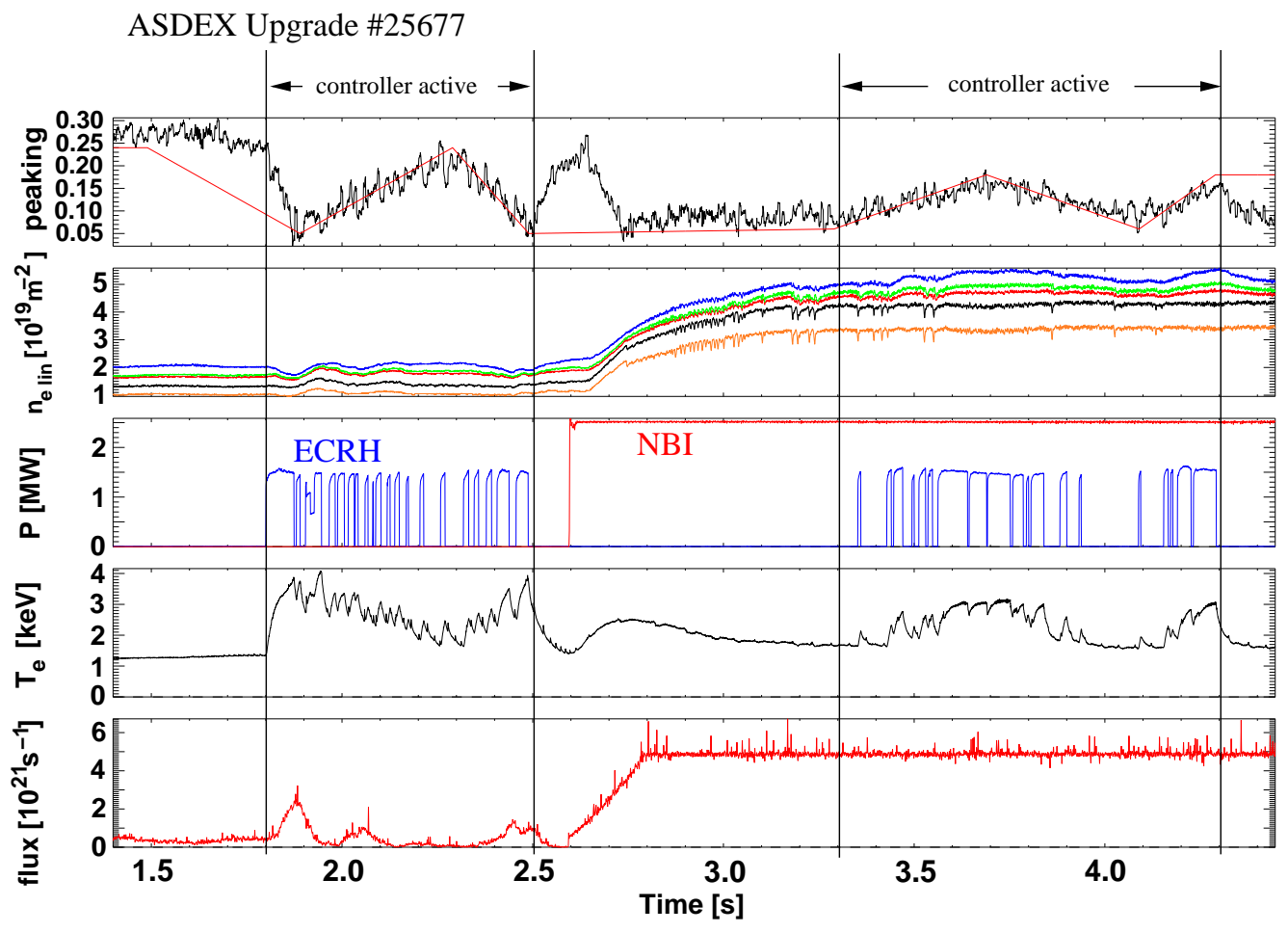

FIG. 9: Feedback control of the density peaking in L-mode and H-mode in one discharge: The controller was active in the two marked time windows. The diagram shows the time traces like in figure 8, where the third box in addition contains the NBI power trace. The top to bottom order of the interferometer time traces is the same as in figure 8 .

As an increasing number of diagnostics on ASDEX Upgrade is currently being upgraded to provide real-time capabilities, real-time density profiles may also include data from other diagnostics such as Thomson scattering, lithium beam impact excitation spectroscopy or reflectometry in the near future. Due to the installation of new gyrotrons, the available ECRH power on ASDEX Upgrade will increase by a factor of 2-3 by the end of 2010 an thus extend the possibilities of modifying the density profile.

As the preparatory experiments contain a large number of heating pulses injected into the plasma at different global parameters, they provide a good basis for the more detailed analysis of the coupling mechanism between temperature and density profile. Whereas the studies on the density response to central heating that can be found in literature do usually focus on the profiles that occur at different levels of heating power after a sufficiently long settling time, the set of heating pulses in our preparatory experiments yields the opportunity of study- ing the transient behaviour of the density when ECRH is switched on and off. We expect that the comparison of the experimentally observed response to sudden changes of the heating power and numerical transport simulations will provide deeper insight into the coupling between density and temperature profile.

Besides active control of the density profile, there are further applications of a real-time density profile. This profile is an important input to ray-tracing algorithms which numerically predict the trajectory of the ECRH microwave beam in the plasma. On ASDEX Upgrade, a real-time feedback system for active suppression of neoclassical tearing modes (NTMs) is currently being built up [16], which is aiming at detecting NTMs in the plasma and stabilizing them by local electron cyclotron current drive (ECCD). For this purpose, the ECRH launcher has to be steered in a way that power deposition and current drive occur at the flux surface that corresponds to the mode location. This requires real-time ray tracing and thus knowledge of the density profile.
[1] C. Mazotta, O. Tudisco, A. Canton, P. Innocente, M. DeBenedetti, E. Giovannozzi, D. Marocco, P. Micozzi, G. Monari, G. Rocchi, Physica Scripta T123 79-83
(2006).

[2] K. McCormick, S. Fiedler, G. Kocsis, J. Schweinzer, S. Zoletnik, Fusion Engineering and Design T34-35 125-134 
(1997).

[3] G.T. Hoang, C. Bourdelle, B. Pegurie, B. Schunke, J.F. Artraud, J. Bucalossi, F. Clairet, C. Fenzi-Bonizec, X. Garbet, C. Gil, R. Guirlet, F. Imbeaux, J. Lasalle, T. Loarer, C. Lowry, J.M. Travere, E. Tsitrone, and Tore Supra team, Physical Review Letters 90155002 (2003).

[4] C. Angioni, A.G. Peeters, X. Garbet, A. Manini, F. Ryter, and ASDEX Upgrade Team, Nuclear Fusion 44 827845 (2004).

[5] L. Zabeo, A. Murari, E. Joffrin, D. Mazon, C. Talierco, Plasma Physics and Controlled Fusion 44 24832494 (2002).

[6] F. Saint-Laurent, P. Houy, G. Martin, D. Moreau, D. Moulin, $26^{\text {th }}$ EPS Conference on Controlled Fusion and Plasma Physics, Europhysics Conference Abstracts Vol.23J 1033-1036 (1999).

[7] R. Fischer, C. Fuchs, B. Kurzan, W. Suttrop, E. Wolfrum, and ASDEX Upgrade Team, Fusion Science and Technology 58 675-684 (2010).

[8] W. Schneider, P. McCarthy, K. Lackner, O. Gruber, K. Behler, P. Martin, R. Merkel, Fusion Engineering and Design 48 127-134 (2000).

[9] L. Giannone, W. Schneider, P. McCarthy, A. Sips, W. Treutterer, K. Behler, T. Eich, J. Fuchs, N. Hicks, A. Kallenbach, M. Maraschek, A. Mlynek, G. Neu, G. Pautasso, G. Raupp, M. Reich, K. Schuhbeck, J. Stober, F. Volpe, T. Zehetbauer, ASDEX Upgrade Team, M. Cerna, T. Debelle, B. Marker, S. McCaslin, M. Munroe,
L. Wenzel, Fusion Engineering and Design 84 825-828 (2009).

[10] A. Mlynek, G. Schramm, H. Eixenberger, G. Sips, K. McCormick, M. Zilker, K. Behler, J. Eheberg, and ASDEX Upgrade Team, Review of Scientific Instruments $\mathbf{8 1}$ 033507 (2010).

[11] W. Treutterer, G. Neu, G. Raupp, T. Zehetbauer, D. Zasche, K. Lüddecke, R. Cole, ASDEX Upgrade Team, Fusion Engineering and Design 85 466-469 (2010).

[12] R.M. Gilgenbach, M.E. Read, K.E. Hackett, R. Lucey, B. Hui, V.L. Granatstein, K.R. Chu, A.C. England, C.M. Loring, O.C. Eldridge, H.C. Howe, A.G. Kulchar, E. Lazarus, M. Murakami, J.B. Wilgen, Physical Review Letters 44647 (1980).

[13] H. Weisen, I. Furno, TCV Team, Nuclear Fusion 411227 (2001).

[14] C. Angioni, E. Fable, M. Greenwald, M. Maslov, A.G. Peeters, H. Takenaga, H. Weisen, Plasma Physics and Controlled Fusion 51124017 (2009).

[15] F. Ryter, C. Angioni, A.G. Peeters, F. Leuterer, H.-U. Fahrbach, W. Suttrop, ASDEX Upgrade Team, Physical Review Letters 95085001 (2005).

[16] M. Reich. K. Behler, R. Drube, L. Giannone, A. Kallenbach, A. Mlynek, J. Stober, W. Treutterer, ASDEX Upgrade Team, Fusion Science and Technology 58 727-732 (2010). 